\title{
Epigenome-wide base-resolution profiling of DNA methylation in chorionic villi of fetuses with Down syndrome by methyl- capture sequencing
}

Ji Hyae Lim', ${ }^{1,2}$ Yu-Jung Kang ${ }^{1}$, Bom Yi Lee ${ }^{3}$, You Jung Han', Jin Hoon Chung ${ }^{4}$, Moon Young Kim4, Min Hyoung $\mathrm{Kim}^{5}$, Jin Woo Kim${ }^{6}$, Youl-Hee Cho ${ }^{2^{*}}$ and Hyun Mee Ryu ${ }^{1,7^{*}}$

\begin{abstract}
Background: Epigenetic mechanisms provide an interface between environmental factors and the genome and are influential in various diseases. These mechanisms, including DNA methylation, influence the regulation of development, differentiation, and establishment of cellular identity. Here, we performed high-throughput methylome profiling to determine whether differential patterns of DNA methylation correlate with Down syndrome (DS).

Materials and methods: We extracted DNA from the chorionic villi cells of five normal and five DS fetuses at the early developmental stage (12-13 weeks of gestation). Methyl-capture sequencing (MC-Seq) was used to investigate the methylation levels of $\mathrm{CpG}$ sites distributed across the whole genome to identify differentially methylated CpG sites (DMCs) and regions (DMRs) in DS. New functional annotations of DMR genes using bioinformatics tools were predicted.
\end{abstract}

Results: DNA hypermethylation was observed in DS fetal chorionic villi cells. Significant differences were evident for 4,439 DMCs, including hypermethylation $(n=4,261)$ and hypomethylation $(n=178)$. Among them, 140 hypermethylated DMRs and only 1 hypomethylated DMR were located on 121 genes and 1 gene, respectively. One hundred twenty-two genes, including 141 DMRs, were associated with heart morphogenesis and development of the ear, thyroid gland, and nervous systems. The genes were significantly associated with DS and various diseases, including hepatopulmonary syndrome, conductive hearing loss, holoprosencephaly, heart diseases, glaucoma, and musculoskeletal abnormalities.

Conclusions: This is the first study to compare the whole-epigenome DNA methylation pattern of the chorionic villi cells from normal and DS fetuses at the early developmental-stage using MC-seq. Overall, our results indicate that the chorionic villi cells of DS fetuses are hypermethylated in all autosomes and suggested that altered DNA methylation may be a recurrent and functionally relevant downstream response to DS in human cells. This study provides basic information for future research focused on the pathophysiology of the DS and its potential effects, as well as the role DNA methylation plays in the early developmental stage of DS fetuses.

Keywords: Down syndrome, Epigenetics, DNA methylation, Chorionic villi, Methyl-capture sequencing

\footnotetext{
* Correspondence: yhcho@hanyang.ac.kr; hmryu@yahoo.com

${ }^{2}$ Department of Medical Genetics, College of Medicine, Hanyang University,

222, Wangsimni-ro, Seongdong-gu, Seoul 04763, Republic of Korea

${ }^{1}$ Center for Biomarker Research and Precision Medicine, CHA Advanced

Research Institute, Gyeonggi-do, Republic of Korea

Full list of author information is available at the end of the article
}

(c) The Author(s). 2019 Open Access This article is distributed under the terms of the Creative Commons Attribution 4.0 International License (http://creativecommons.org/licenses/by/4.0/), which permits unrestricted use, distribution, and reproduction in any medium, provided you give appropriate credit to the original author(s) and the source, provide a link to the Creative Commons license, and indicate if changes were made. The Creative Commons Public Domain Dedication waiver (http://creativecommons.org/publicdomain/zero/1.0/) applies to the data made available in this article, unless otherwise stated. 


\section{Background}

In the postgenomic era, genetic-epigenetic interactions have become a major focus. There are two main types of these interactions: cis interactions involve short-range effects of single-nucleotide polymorphisms and haplotypes; and trans interactions involve the effects of chromosomal aneuploidy on DNA methylation of $\mathrm{CpG}$ sites [1]. Trans-acting epigenetic effects of chromosome gain or loss lead to abnormal development and embryonic lethality. However, fetuses with Down syndrome (DS), known as the most common numerical chromosomal abnormality, survive to term. Therefore, the trans-acting epigenetic effects of DNA methylation are attracting increasing attention in DS research.

The estimated incidence of DS, the most prevalent genetic cause of developmental disabilities, is generally between 1 in 1000 to 1 in 1100 live births worldwide according to the World Health Organization [2]. It is characterized by over 80 clinical features that include mental retardation, stereotypical facial features, poor muscle tone, and short stature of various penetrance, and is associated with an increased risk of congenital heart disease, diabetes, leukemia, and other diseases [3]. The changes associated with DS begin early in fetal development because it is a congenital abnormality caused by the presence of an additional whole or partial copy of human chromosome 21 (HSA21). Therefore, theoretically, DS results in a 1.5-fold increased expression of genes on HSA21 and maintains genomic balance in genes on the other chromosomes. However, transcriptome analyses in DS of humans and mouse models have not revealed a strong linear correlation between the genomic imbalance of HSA21 and gene expression levels [4-8]. Transcriptomes of fetal fibroblasts from a monozygotic twin pair discordant for DS has suggested that differentially expressed genes are organized in large chromosomal domains [9]. In our prior transcriptome analysis using chorionic villi cells in the early developmental stage of DS fetuses, we also reported gene expression changes on all chromosomes, not on only HSA21 [10]. These results suggest that genes are embedded in temporally and spatially highly coordinated regulatory networks. The functional consequences of epigenetic changes, which occur at a much higher rate than DNA sequence changes $(4.46 \times 10-4$ versus $7 \times$ 10-9) [11], may significantly contribute to the clinical phenotype variation of DS.

In the early development stage of a DS fetus (the first or second trimester of pregnancy), a set of differentially methylated regions (DMRs) was recently demonstrated not to be enriched for HSA21 genes. Rather, these DMRs were roughly evenly distributed on all chromosomes [12-15]. Moreover, global DNA hypermethylation was demonstrated in the fetal DS cortex and placenta, with only a partial overlap of DMRs between these cell types [14, 15]. Epigenome-wide profiling in DS-based DNA methylation microarray has been performed in several tissues, including leukocytes, skin fibroblasts, buccal cells, blood, placenta, and brain [14-19]. However, these methods suffer from low genome coverage (approximately $1-3 \%$ of all $\mathrm{CpG}$ sites in the human genome) and introduce errors by probe cross-hybridization [20].

Here, we investigated the epigenome-wide DNA methylation patterns in the chorionic villi cells of euploid and DS fetuses using high-resolution methylcapture sequencing (MC-seq) at a single-base resolution, which covered approximately 3.2 million $\mathrm{CpG}$ sites in the human genome and identified new DMRs in DS. New functional annotations of genes, including DMRs, were suggested using various bioinformatics tools.

\section{Results}

Overview of MC-Seq analysis of human chorionic villi cells All chorionic villi cells were obtained in the first trimester of pregnancy (12 13 weeks of gestation, Additional file 1: Table S1). At the time of chorionic villus sampling, there were no significant differences between DS and normal groups regarding maternal age, gestational age, nuchal translucency, and gender ratio of the fetuses ( $p>0.05$ for all, Additional file 1: Table S1).

MC-seq was used to quantify DNA methylation in various CpG sites in chorionic villi cells of DS and normal fetuses. On average, 3,103,571 CpG sites with a sequencing depth $>300$ in each of 10 chorionic villi samples (5 DS and 5 normal samples, matching gestational age, and fetal sex) were analyzed (Additional file 1: Table S2). Assayed CpG sites represented approximately $11 \%$ of all 28,217 , 449 CpG sites in the human genome (hg19), spread across regions CpG islands (CGI; 1,597,050 CpGs), CGI shores (defined as $2 \mathrm{~kb}$ upstream or downstream of CGIs; 810, $354 \mathrm{CpGs}$ ), and other genomic regions (732,656 CpGs). These CpGs were distributed in the intragenic region (1, 559,007 CpGs) consisting of $5^{\prime}$ untranslated region (UTR), exonic, intronic, and 3 'UTR, and the intergenic regions (1,581,053 CpGs) including promoters (defined as a transcription start site upstream 2 kb; 820,913 CpGs), downstream (defined as a transcription termination site downstream $1.5 \mathrm{~kb}$; 43,216 CpGs), and other regions (Additional file 1: Table S3).

\section{Higher DNA methylation levels in whole genome of the chorionic villi of fetuses with DS}

We analyzed DNA methylation of DS within a variety of genomic contexts of differentially methylated CpG sites (DMCs) and DMRs (see "Materials and methods" section). In DS samples, hypermethylation and hypomethylation were detected in 4,261 and 178 DMCs, respectively (Fig. 1). The number of hypermethylated DMCs far exceeded the 


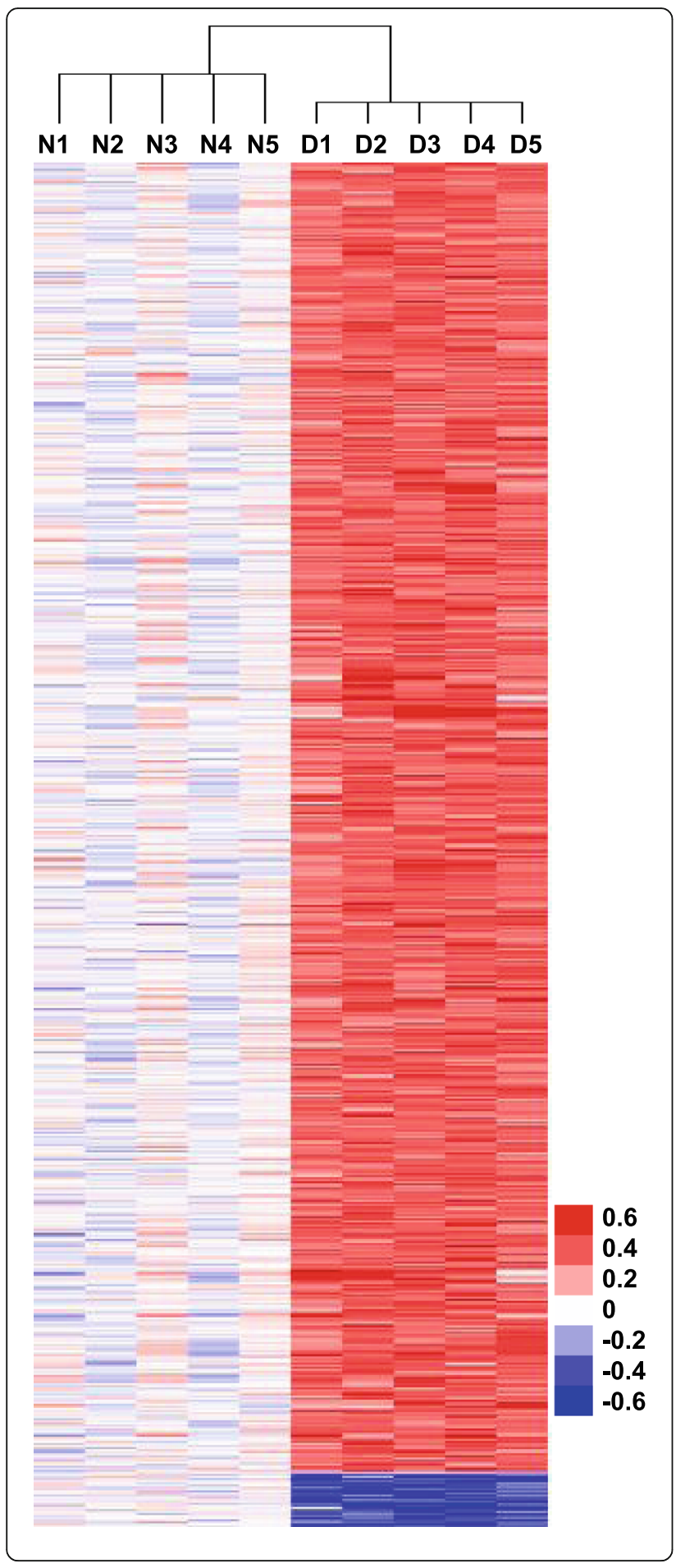

Fig. 1 Hierarchical clustering of DMCs. The methylation degree values from MC-seq, applying independent $t$ test $(p<0.05)$ and a fold-change criterion (|delta _ average of methyl degree $\geq 0.3$ in DS versus normal) produced a set of 4,439 DMCs. The $p$ values were corrected using the Benjamini and Hochberg false discovery rate (FDR) method to control false positive results from multiple testing. The methylation degree values for these DMCs were subjected to hierarchical clustering. Biological samples are on the $x$-axis and DMCs are on the $y$-axis with strong methylation indicated by the red color and weak or absent methylation by the blue color. N, normal, D, Down syndrome

hypomethylated DMCs in each chromosome of DS chorionic villus samples (Table 1). A comparison of the chromosome distribution of DMCs revealed that hypermethylated DMCs were most numerous on chromosome 19. Additionally, the proportional increase of hypermethylated DMCs to hypomethylated DMCs (number of hypermethylated $\mathrm{DMC}$ /number of hypomethylated DMC) was analyzed by chromosome size. The dominance of hypermethylated DMC was pronounced in all chromosomes and was prominent in chromosomes 8, 19, 20, and 21 (Table 1). Analysis of the distribution of DMCs according to the functional genomic regions, the dominance of hypermethylated DMCs over hypomethylated DMCs was seen in all functional genomic regions of DS (Table 2). The dominance of hypermethylated DMCs was most pronounced in the exonic part of intragenic regions and in CGI (Table 2). The average methylation levels of DMCs in promoter, CGI, and CGI shore in DS fetuses were significantly higher in those of normal fetuses (Additional file 1: Table S4).

\section{The common change of DNA methylation observed in fetal DS}

To further investigate the common changes of DNA methylation observed in fetal DS regardless of tissue type, we compared the DNA methylation data from DS fetal brain tissue with these results. A publicly available dataset of fetal brains (GEO \#: GSE73747) was reanalyzed according to the criteria of DMC selection used in this study. In fetal brains with DS, eight DMCs were hypermethylated and only one DMC was hypomethylated, compared to the methylation patterns in normal fetal brains (Additional file 1: Table S5). Of the DMCs of the brains and the chorionic villi of fetal DS, CPT1B on chromosome 22 and TNXB on chromosome 6 were commonly hypermethylated, regardless of tissue type, but involved none of hypomethylated DMCs. To confirm the DNA methylation pattern of $C P T 1 B$, we performed methylation-specific quantitative real-time polymerase chain reaction (PCR). The $\Delta C t$ value of $C P T 1 B$ was significantly lower in the DS than normal (Table 3). It means that the gene was significantly hypermethylated in the chorionic villi cells of DS fetuses, compared with those in normal fetuses. The DNA methylation pattern of the gene was consistent with that of MC-seq. 
Table 1 Distribution of DMCs on chromosome

\begin{tabular}{|c|c|c|c|c|c|c|c|c|c|}
\hline \multirow[t]{2}{*}{ chr. } & \multirow{2}{*}{$\begin{array}{l}\text { Size } \\
(\mathrm{Mb})\end{array}$} & \multicolumn{3}{|c|}{ Hypermethlyated DMCs in DS } & \multicolumn{3}{|c|}{ Hypomethylated DMCs in DS } & \multirow{2}{*}{$\begin{array}{l}\text { Magnification of } \\
\text { hypermethylated } \\
\text { DMCs to } \\
\text { hypomethylated } \\
\text { DMCs }\end{array}$} & \multirow{2}{*}{$\begin{array}{l}\text { Magnification of } \\
\text { hypermethylated } \\
\text { DMCs to } \\
\text { hypomethylated } \\
\text { DMCs based on } \\
\text { chromosome size }\end{array}$} \\
\hline & & $\bar{n}$ & $\begin{array}{l}\text { DMCs for each } \\
\text { chromosome } \\
\text { of total DMCs (\%) }\end{array}$ & $\begin{array}{l}\text { DMCs based on } \\
\text { chromosome } \\
\text { size (\%) }\end{array}$ & $\bar{n}$ & $\begin{array}{l}\text { DMCs for each } \\
\text { chromosome in } \\
\text { total DMCs (\%) }\end{array}$ & $\begin{array}{l}\text { DMCs based } \\
\text { on chromosome } \\
\text { size }(\%)\end{array}$ & & \\
\hline 1 & 248.96 & 399 & 9.4 & 1.6 & 15 & 8.4 & 0.1 & 26.6 & 0.11 \\
\hline 2 & 242.19 & 290 & 6.8 & 1.2 & 11 & 6.2 & 0.0 & 26.4 & 0.11 \\
\hline 3 & 198.3 & 121 & 2.8 & 0.6 & 6 & 3.4 & 0.0 & 20.2 & 0.10 \\
\hline 4 & 190.22 & 145 & 3.4 & 0.8 & 9 & 5.1 & 0.0 & 16.1 & 0.08 \\
\hline 5 & 181.54 & 218 & 5.1 & 1.2 & 11 & 6.2 & 0.1 & 19.8 & 0.11 \\
\hline 6 & 170.81 & 152 & 3.6 & 0.9 & 7 & 3.9 & 0.0 & 21.7 & 0.13 \\
\hline 7 & 159.35 & 246 & 5.8 & 1.5 & 14 & 7.9 & 0.1 & 17.6 & 0.11 \\
\hline 8 & 145.14 & 289 & 6.8 & 2.0 & 4 & 2.2 & 0.0 & 72.3 & $0.50^{*}$ \\
\hline 9 & 138.4 & 93 & 2.2 & 0.7 & 7 & 3.9 & 0.1 & 13.3 & 0.10 \\
\hline 10 & 133.8 & 217 & 5.1 & 1.6 & 7 & 3.9 & 0.1 & 31.0 & 0.23 \\
\hline 11 & 135.09 & 259 & 6.1 & 1.9 & 16 & 9.0 & 0.1 & 16.2 & 0.12 \\
\hline 12 & 133.28 & 167 & 3.9 & 1.3 & 9 & 5.1 & 0.1 & 18.6 & 0.14 \\
\hline 13 & 114.36 & 99 & 2.3 & 0.9 & 4 & 2.2 & 0.0 & 24.8 & 0.22 \\
\hline 14 & 107.04 & 148 & 3.5 & 1.4 & 3 & 1.7 & 0.0 & 49.3 & 0.46 \\
\hline 15 & 101.99 & 156 & 3.7 & 1.5 & 4 & 2.2 & 0.0 & 39.0 & 0.38 \\
\hline 16 & 90.34 & 179 & 4.2 & 2.0 & 13 & 7.3 & 0.1 & 13.8 & 0.15 \\
\hline 17 & 83.26 & 182 & 4.3 & 2.2 & 9 & 5.1 & 0.1 & 20.2 & 0.24 \\
\hline 18 & 80.37 & 93 & 2.2 & 1.2 & 4 & 2.2 & 0.0 & 23.3 & 0.29 \\
\hline 19 & 58.62 & 476 & 11.2 & 8.1 & 15 & 8.4 & 0.3 & 31.7 & $0.54^{*}$ \\
\hline 20 & 64.44 & 133 & 3.1 & 2.1 & 2 & 1.1 & 0.0 & 66.5 & $1.03^{*}$ \\
\hline 21 & 46.71 & 60 & 1.4 & 1.3 & 2 & 1.1 & 0.0 & 30.0 & $0.64^{*}$ \\
\hline 22 & 50.82 & 139 & 3.3 & 2.7 & 6 & 3.4 & 0.1 & 23.2 & 0.46 \\
\hline Total & & 4,261 & 100.0 & & 178 & 100.0 & & & \\
\hline
\end{tabular}

Chr. chromosome, DMCs differentially methylated CpG sites, DS Down syndrome, $M b$ megabases, $n$ number

*The proportional increase of hypermethylated DMCs to hypomethylated DMCs is 0.5 or more, depending on the chromosome size. It means that these chromosomes were more enriched with hypermethylated CpG sites.

\section{Validation of MC-Seq analysis by methylation-specific quantitative real-time PCR}

We selected 141 DMRs from the total of 4439 DMCs based on criteria for DMR selection (Additional file 1: Table S6). All DMRs, excluding one, were hypermethylated in the whole genome. We selected the top five DMRs (ZNF837, C11orf95, KIAA1875, SHROOM1, and CASZ1) and confirmed their DNA methylation patterns using methylation-specific quantitative realtime PCR. The DNA methylation patterns of the DMRs were consistent with that of MC-seq (Table 3). The largest number of DMRs was present on chromosome 19, as observed for DMCs (Additional file 1: Table S7). Most hypermethylated DMRs were presented in the exonic region of intragenic regions (Fig. 2). One hundred twenty-two genes including DMRs were used for the functional annotation analysis related to DS.
Significant influence of DMRs in biological process and disease associations of DS

In the in silico analysis using the 122 DMR genes, gene ontology (GO) annotation and disease association analyses were performed using a statistical hypergeometric test, with a significance level of $p<0.05$ and a minimum of three genes. In the biological process category of GO annotation (Table 4), regulation of heart morphogenesis was the most significant $(\operatorname{adj} P=0.0050)$. Related DMR genes were $S L X 1$, WNT3A, TBX1, and SOX9. The largest number of DMR genes $(n=34)$ involved in system development $(\operatorname{adj} P=0.0084)$. Significant correlations were evident for the development of the ear, thyroid gland, and nervous system $(\operatorname{adj} P<0.05$ for all). The glutamate receptor signaling pathway and regulation of ion transport were also significantly related to the DMR genes $(\operatorname{adj} P<0.05$ for both). In the molecular function category of GO annotation (Table 4), KCNA5, SHANK1, and CACNA1H were 
Table 2 Distribution of DMCs on functional genomic regions

\begin{tabular}{|c|c|c|c|c|c|}
\hline \multirow{2}{*}{$\begin{array}{l}\text { Functional } \\
\text { genomic } \\
\text { regions }\end{array}$} & \multicolumn{2}{|c|}{ Hypermethlyated DMCs } & \multicolumn{2}{|c|}{ Hypomethylated DMCs } & \multirow{2}{*}{$\begin{array}{l}\text { Magnification of } \\
\text { hypermethylated } \\
\text { DMCs to } \\
\text { hypomethylated } \\
\text { DMCs }\end{array}$} \\
\hline & $n$ & $\%$ & $n$ & $\%$ & \\
\hline Intragenic & 2,539 & 57.2 & 100 & 2.3 & 25.4 \\
\hline $5^{\prime} U T R$ & 98 & 2.2 & 2 & 0.0 & 49.0 \\
\hline $3^{\prime} U T R$ & 146 & 3.3 & 10 & 0.2 & 14.6 \\
\hline Intronic & 905 & 20.4 & 66 & 1.5 & 13.7 \\
\hline Exonic & 1,390 & 31.3 & 22 & 0.5 & 63.2 \\
\hline Intergenic & 1,216 & 27.4 & 49 & 1.1 & 24.8 \\
\hline Promoter & 488 & 11.0 & 22 & 0.5 & 22.2 \\
\hline Downstream & 60 & 1.4 & 0 & - & - \\
\hline CGl & 3,053 & 68.8 & 59 & 1.3 & 51.7 \\
\hline CGI shore & 499 & 11.2 & 23 & 0.5 & 21.7 \\
\hline
\end{tabular}

CGI CpG island, DMCs differentially methylated CpG sites, UTR untranslated region

Percentages of DMCs on functional genomic regions was measured based on total 4439 DMCs

most significantly associated with the scaffold protein binding $(\operatorname{adj} P=0.0143)$. The largest number of genes $(n=$ 13) was involved in sequence-specific DNA binding transcription factor activity $(\operatorname{adj} P=0.0388)$. However, there was no significant association with the cellular component category of GO annotation. The disease associations of the DMR genes are shown in Table 5. The most statistically significant associations were for hepatopulmonary syndrome and conductive hearing loss (for both; $\operatorname{adj} P=$ $0.0039)$. The largest number of genes $(n=7)$ was involved in congenital abnormalities (adj $P=0.0224)$. DMR genes were also significantly associated with DS, craniofacial

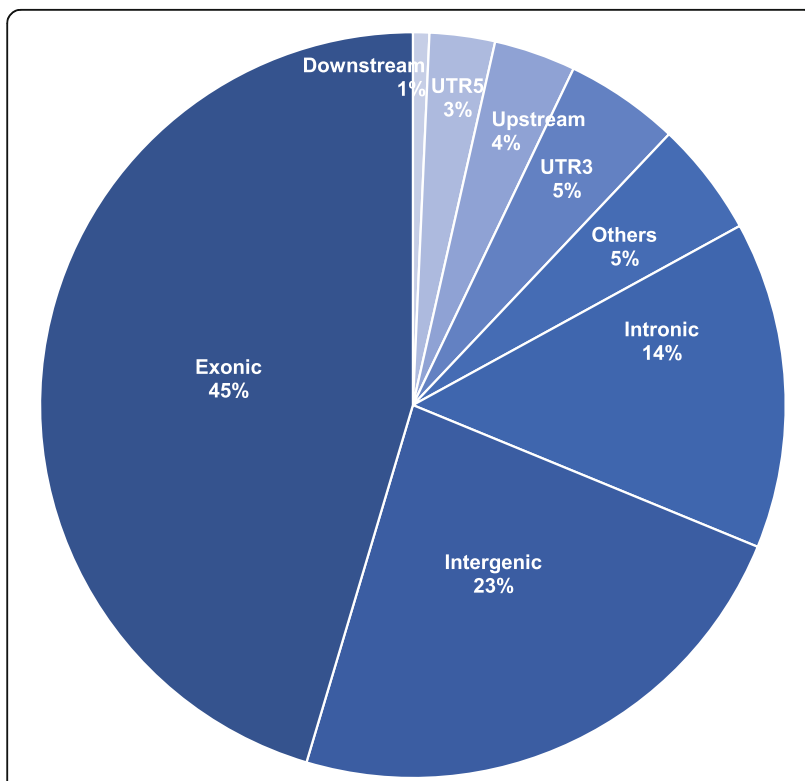

Fig. 2 Distribution of DMRs according to the functional genomic regions abnormality, heart disease, chromosome aberrations, musculoskeletal abnormalities, and genetic translocation (adj $P$ $<0.05$ for all).

\section{Discussion}

The mechanisms underlying congenital impairment in DS are unclear. Accumulating evidence suggests that DS phenotypes, including various pathological changes, occur by alterations of the complex regulation of many genes on and outside HSA21. The alterations could affect the roles of the upstream effector genes, located on HSA21, and the downstream target genes that are affected by DNA methylation, which are distributed throughout the genome of DS individuals. Therefore, investigating the epigenomic changes that contribute to the various phenotypes of DS may improve our understanding of the pathophysiology of DS.

In the current study, we performed DNA methylation profiling at single-base resolution in the chorionic villi cells of human DS and euploid fetuses at an early developmental stage. Global DNA hypermethylation across the whole

Table $3 \mathrm{Ct}$ values of genes using methylation specific quantitative real-time PCR

\begin{tabular}{|c|c|c|c|c|c|c|c|}
\hline \multirow{2}{*}{$\begin{array}{l}\text { Target } \\
\text { gene }\end{array}$} & \multicolumn{3}{|c|}{$N(n=10)$} & \multicolumn{3}{|l|}{$\mathrm{DS}(n=8)$} & \multirow[t]{2}{*}{$p$ value } \\
\hline & $\overline{\operatorname{MSRE}(-)}$ & MSRE (+) & $\Delta \mathrm{Ct}$ & MSRE ((-) & MSRE (+) & $\overline{\Delta C t}$ & \\
\hline CPT1B & 32.73 & 35.31 & 2.58 & 33.35 & 33.55 & 0.19 & 0.033 \\
\hline ZNF837 & 35.47 & 37.59 & 2.13 & 36.77 & 36.61 & 0.21 & 0.033 \\
\hline C11orf95 & 29.62 & 33.74 & 4.12 & 30.02 & 30.74 & 0.72 & 0.006 \\
\hline KIAA1875 & 30.55 & 41.94 & 11.59 & 34.37 & 35.65 & 2.89 & 0.007 \\
\hline SHROOM1 & 30.07 & 34.12 & 4.05 & 29.86 & 30.73 & 0.86 & 0.001 \\
\hline CASZ1 & 34.00 & 37.83 & 3.27 & 34.33 & 35.19 & 0.85 & 0.008 \\
\hline
\end{tabular}


Table 4 GO analysis of identified DMR genes

\begin{tabular}{|c|c|c|c|c|}
\hline & Pathway & GeneSymbol & rawP & $\operatorname{adjP}$ \\
\hline \multirow[t]{13}{*}{$\mathrm{BP}$} & Regulation of heart morphogenesis & SIX1 WNT3A, TBX1, SOX9 & $5.63 e-06$ & 0.0050 \\
\hline & Glutamate receptor signaling pathway & MINK1, GRIN3B, SHANK1, MAPK8IP2 & 0.0001 & 0.0074 \\
\hline & Organ morphogenesis & $\begin{array}{l}\text { SIX1, LEFTY1, SIX6, TBX1, SOX9, AXIN2, } \\
\text { DUOX2, UNCX, WNT3A, TLX1, F7, MYO15A, } \\
\text { FOXL1, KIF26B }\end{array}$ & 0.0001 & 0.0074 \\
\hline & Nervous system development & $\begin{array}{l}\text { MINK1, SLC8A3, SOX9, CACNA1H, STMN3, UNCX, } \\
\text { TLX1, SEPP1, FOXL1, MAPK8IP2, FOXD2, RTN1, SIX1, } \\
\text { TACC2, TBX1, SHANK1, MNX1, DUOX2, SALL3, WNT3A, } \\
\text { BRSK2, CRMP1, PURA }\end{array}$ & $3.20 \mathrm{e}-05$ & 0.0074 \\
\hline & Anatomical structure morphogenesis & $\begin{array}{l}\text { MINK1, LEFTY1, SIX6, SOX9, SHROOM1, CACNA1H, } \\
\text { UNCX, TLX1, MYO15A, FOXL1 MAPK8IP2, FOXD2, } \\
\text { KIF26B, RSG1, SIX1, TBX1, SHANK1, MNX1, AXIN2, } \\
\text { DUOX2, SALL3, ECE1, WNT3A, BRSK2, F7, CRMP1 }\end{array}$ & $2.06 \mathrm{e}-05$ & 0.0074 \\
\hline & Central nervous system development & $\begin{array}{l}\text { SIX1, SLC8A3, TACC2, TBX1, MNX1, SOX9, DUOX2, UNCX, } \\
\text { SALL3, WNT3A, TLX1, SEPP1, FOXL1 }\end{array}$ & $8.41 e-05$ & 0.0074 \\
\hline & Ear development & DUOX2, SIX1, ECE1, WNT3A, MYO15A, TBX1, SOX9 & $6.29 \mathrm{e}-05$ & 0.0074 \\
\hline & System development & $\begin{array}{l}\text { MINK1, LEFTY1, SIX6, SOX9, CACNA1H, TLX1, AHSP, SEPP1, } \\
\text { RTN1, SIX1, ZNF354A, CBFA2T3, TACC2, SHANK1, MNX1, } \\
\text { AXIN2, SALL3, ECE1, WNT3A, SLC8A3, STMN3, UNCX, MYO15A, } \\
\text { FOXL1, MAPK8IP2, KIF26B, FOXD2, TBX1, DUOX2, HLA-DOA, } \\
\text { BRSK2, CRMP1, F7, PURA }\end{array}$ & 0.0002 & 0.0084 \\
\hline & Thyroid gland development & DUOX2, SIX1, TBX1 & 0.0002 & 0.0084 \\
\hline & Otic vesicle development & SIX1, TBX1, SOX9 & 0.0002 & 0.0084 \\
\hline & Inner ear morphogenesis & SIX1, WNT3A, MYO15A, TBX1, SOX9 & 0.0002 & 0.0084 \\
\hline & Inner ear development & DUOX2, SIX1, WNT3A, MYO15A, TBX1, SOX9 & 0.0002 & 0.0084 \\
\hline & Regulation of ion transport & KCNA5, MINK1, DPP6, GRIN3B, SHANK1, MAPK8IP2, BEST1 & 0.0006 & 0.0133 \\
\hline \multirow[t]{3}{*}{ MF } & Scaffold protein binding & KCNA5, SHANK1, CACNA1H & 0.0001 & 0.0143 \\
\hline & $\begin{array}{l}\text { RNA polymerase II distal enhancer } \\
\text { sequence-specific DNA binding } \\
\text { transcription factor activity }\end{array}$ & PURA, FOXL1, SOX9, FOXD2, NFIX & 0.0002 & 0.0143 \\
\hline & Sequence-specific DNA binding transcription factor activity & $\begin{array}{l}\text { SIX1, ZNF354A, CBFA2T3, SIX6, TBX1, MNX1, SOX9, UNCX, } \\
\text { TLX1, PURA, FOXL1, FOXD2, NFIX }\end{array}$ & 0.0019 & 0.00388 \\
\hline
\end{tabular}

$B P$ biological process, $M F$ molecular function, $\operatorname{raw} P p$ value from hypergeometric test, adjP $p$ value adjusted by the multiple test adjustment

epigenome was evident in DS fetuses, with methylation differing in 4439 DMCs compared to that in euploid fetuses. Most DMCs were hypermethylated in DS and only approximately $4 \%$ of DMCs were hypomethylated. These DMCs were distributed in all chromosomes, regardless of the methylation pattern in DS. Hypermethlyated DMCs were pronounced in the exonic regions in intragenic regions and the promoters in intergenic regions. Additionally, two genes $(C P T 1 B$ and $T N X B)$ were commonly hypermethylated in fetal brain and chorionic villi in DS. We identified 141 DMRs that ranged from three to 79 DMCs. All DMRs, except one DMR, were hypermethylated in DS. In a functional annotation of identified DMR genes, we found that these genes with hypermethylated DMRs were significantly related to the various phenotypes and pathophysiology including chromosomal aberration and congenital abnormalities of DS. This finding suggests the possibility of fetal epigenetic therapy by inhibiting the global DNA methylation in DS. Furthermore, chorionic villi cells (e.g., trophoblasts) are the major origin of cell-free fetal DNA in maternal blood [21]. Therefore, profiling DNA methylation in chorionic villi cells of fetal DS could lead to potential clinical application to develop novel approaches for the noninvasive prenatal test of DS in the first trimester of pregnancy by analysis of fetal DS-specific DNA methylation in maternal blood.

Global hypermethylation in DS was conserved in different tissue types and developmental stages [13-19]. However, the number and location of DMCs differed in the whole genome depending on tissue types and developmental stage. In peripheral blood leukocytes of DS adults, eight genes (TMEM131, TCF7, CD3Z/CD247, SH3BP2, EIF4E, PLD6, SUMO3, and CPT1B) were hypermethylated [18]. In the fetal cortex of DS, nine genes $(C 1$ orf $35, C P T 1 B, D E C R 2$, FAM83H, GLI4, LRRC14, LRRC24, STK19, and TNXB) were hypermethylated [14]. In the fetal placenta of DS, hypermethylation of $C P T 1 B, T C F 7$, and FAM62C was evident [15]. We also found hypermethylation in the 5'UTR 
Table 5 Disease association of identified DMR genes

\begin{tabular}{|c|c|c|c|}
\hline Disease & GeneSymbol & rawP & adjp \\
\hline Hepatopulmonary syndrome & KCNA5, DUOX2, ECE1, THBD & $8.40 \mathrm{e}-05$ & 0.0039 \\
\hline Hearing loss, conductive & TECTA, SIX1, MYO15A, TBX1 & $8.01 e-05$ & 0.0039 \\
\hline Holoprosencephaly & SIX6, GDF1, MNX1 & 0.0002 & 0.0062 \\
\hline Craniofacial abnormalities & WNT3A, TBX1, GDF1, SOX9, AXIN2 & 0.0011 & 0.0187 \\
\hline Down syndrome & RAB9BP1, SUMO3, ANKRD2OA11P, HLA-DOA, EFEMP2 & 0.0011 & 0.0187 \\
\hline Heart diseases & KCNA5, F7, TBX1, GDF1, THBD & 0.0033 & 0.0224 \\
\hline Chromosome aberrations & TLX1, CBFA2T3, TBX1, MNX1, SOX9 & 0.0035 & 0.0224 \\
\hline Testicular diseases & ALPPL2, YJEFN3, SOX9 & 0.0027 & 0.0224 \\
\hline Congenital abnormalities & SIXI, SIX6, TBXI, GDF1, MNX1, SOX9, AXIN2 & 0.0019 & 0.0224 \\
\hline Hearing loss, non-syndromic & TECTA, SIX1, MYO15A & 0.0029 & 0.0224 \\
\hline Glaucoma & $S I X 1, S I X 6, B E S T 1$ & 0.0021 & 0.0224 \\
\hline Musculoskeletal abnormalities & TBX1, GDF1, MNX1, SOX9, AXIN2 & 0.0020 & 0.0224 \\
\hline Translocation, genetic & TLX1, SLCBA3, CBFA2T3, MNX1, SOX9 & 0.0065 & 0.0290 \\
\hline
\end{tabular}

of $C P T 1 B$ in chorionic villi cells at an early developmental stage of fetal DS. These results suggest that hypermethylation of $C P T 1 B$ is conserved in various tissue samples of the different developmental origin of DS and may be a common epigenetic characteristic in DS. CPT1B on chromosome 22 is one of the carnitine palmitoyltransferase 1 genes [22]. This enzyme is required for the net transport of longchain fatty acyl-CoAs from the cytoplasm to the mitochondria $[14,22]$. Mitochondrial dysfunction has been found in the brains of patients with various psychiatric disorders, including DS [14]. Placental mitochondrial dysfunction may also be critical in a range of gestational disorders, which has important implications for maternal and fetal/offspring health. Therefore, these results provide the new insight that the hypermethylation of $C P T 1 B$ may be associated with mitochondrial dysfunction in the developing DS.

In this study, the methylation degree of ZNF837, C11orf95, KIAA1875, SHROOM1, and CASZ1 with more than 40 hypermethylated DMCs were confirmed by methylation-specific real-time PCR. The results were consistent with the MC-seq results. Among these hypermethylated DMR genes, SHROOM1 and CASZ1 appear important potentials in pathophysiology of complications related to DS. SHROOM1 on chromosome 5 encode Shroom Family Member 1. SHROOM family members play diverse roles in the development of the nervous system and other tissues and may be involved in the assembly of microtubule arrays during cell elongation [23]. Diseases associated with SHROOM1 include atrial septal defect 2 and atrial heart septal defect. GO annotations related to this gene include actin filament binding. Ectopic expression of SHROOM1 elicited apical accumulation of gamma-tubulin in naive epithelial cells, consistent with a role for SHROOM1 in governing microtubule architecture [24]. It was recently reported that patients with knee osteoarthritis had marked hypermethylation status in all SHROOM1 differentially methylated sites between damaged and non-damaged tissues [25]. The authors suggested hypermethylated SHROOM1 as a promising candidate for functional studies of osteoarthritis pathology [25]. In another study, hypermethylation of SHROOM1 was present in DS fetuses with or without congenital heart defects, as well as in fetuses with heart malformations [16]. Congenital heart defects are the most common malformation occurring in DS. There is also an increased risk of skeletal abnormalities, including osteoarthritis, in DS. There certainly is a higher incidence of heart and joint problems in DS. However, these disorders are thought to have a multifactorial etiology and the main causes are largely unknown. Therefore, hypermethylation of SHROOM1 in DS may be potentially associated with the pathophysiology of the congenital heart defects and osteoarthritis related to DS. Additionally, CASZ1 on chromosome 1 is a proteincoding gene of castor zinc finger 1 , which is a zinc finger transcription factor. The single-nucleotide polymorphisms in this gene are associated with blood pressure variation. Alternative splicing yields multiple transcript variants encoding different protein isoforms [26]. They are involved in vascular assembly and morphogenesis through direct transcriptional regulation of EGFL7 [27]. Diseases associated with CASZ1 include retroperitoneal sarcoma and retroperitoneum carcinoma. Interestingly, Casz1 is expressed in a number of developing tissues, including neural, endothelial, and cardiac tissues. Improper regulation of CASZ1 could potentially lead to the defects that are collectively observed in DS, such as cognitive defects, congenital heart defects, and hypertension $[28,29]$. CASZ1 also has an established role in regulating cellular adhesion in multiple tissues. In previous 
studies of CASZ1 function, HSA21 resident-congenital heart disease 5 protein (CHD5) was demonstrated as being interactive and essential protein for CASZ1 function, with the CHD5-CASZ1 interaction being necessary for cardiac morphogenesis [30,31]. Therefore, a potential mechanism that could link the misregulation of CASZ1 to one or more pathologies related to DS has been suggested. However, the precise mechanism by which CASZ1 is regulated by its interaction with CHD5 has yet to be determined. In this study, we firstly found that exonic region of Casz1 is hypermethylated in DS. This epigenetic change of Casz1 in DS may provide additional insight into the phenotypes generated by its misregulation. Furthermore, ZNF837 on chromosome 19 encodes zinc finger protein 837 and is commonly hypermethylated in the adult and fetal brain, and the fetal placenta in DS [1]. In this study, ZNF837 was identified as the DMR gene with the largest DMCs in the chorionic villi cells in the early developmental stage of DS fetuses. Although this gene may be involved in transcriptional regulation, since ZNF837 is located in the nucleus, an obvious function is still unclear. C11orf95 (Chromosome 11 Open Reading Frame 95) is associated with ependymoma as tumors of the brain and spinal cord [32]. C11orf95-RELA fusion protein translocates spontaneously to the nucleus to activate nuclear factor-kappa B target genes, and rapidly transform neural stem cellsthe originating cells of ependymoma-to form these tumors [33]. However, ependymoma in DS has only been reported so far in only two cases, and the association of ependymoma with DS is a rarity [34, 35]. Finally, KIAA1875 (previous HGNC symbols for WDR97 and encode WD Repeat Domain 97) has an unknown function and protein-coding potential [36]. Further research is needed to understand the epigenetic function and mechanisms of known and still-undiscovered DMR genes. Our results have potential utility value as basic information for further studies of these DMR genes in DS.

Several studies have compared the DNA methylation profiles between normal and DS patients and have demonstrated common epigenetic characteristics. These include global hypermethylation and a lack of enrichment on HSA21. This DNA methylation pattern may be tissue and developmental stage-specific in DS. Moreover, DS as a congenital aneuploidy may involve continuous epigenetic changes in whole genome from embryos early in development. These early epigenetic changes may be the basis of the developmental defects in DS. Therefore, it is important to investigate epigenetic changes in the early development of DS fetuses to clarify the pathogenesis of DS. Recently, global hypermethylation was demonstrated in the fetal cortex and placenta of human DS $[14,15]$. We also found the same methylation pattern in early chorionic villi cells of fetal sex and gestational age-matched DS and normal fetuses. However, the percentage of hypermethylated DMCs identified in each study varied from 1.85 to $4 \%$. The variation likely reflects the impact of various factors, such as tissue type, gestational age at sampling, criteria for DMC selection, and methods used. In this study, we used MCSeq for high-throughput methylome profiling in DS. This is a next-generation sequencing approach that targets specific loci in the epigenome. MC-seq utilizes target- specific bait sequences, circumventing this restriction through bait design and allows for epigenome-wide surveying of specific genomic loci of physiological and clinical interest. Thus, it presents an attractive, cost-effective alternative to uncover novel disease-associated genomic loci. Moreover, MC-seq overcomes the limitations of lower genome coverage in a methylation microarray, high cost, and processing time in whole-genome bisulfite sequencing, while avoiding overrepresentation in next-generation sequencing platforms, such as reduced representation bisulfite sequencing and methylated DNA immunoprecipitation sequencing [20]. Furthermore, in this study, the MC-seq with a depth exceeding $300 \times$ was performed to overcome the technical variation in detecting small inter-individual/inter-group differences. Therefore, we were available to interrogate DNA methylation more precisely in clinical samples at the single-base resolution. However, many of our results were based on databases of bioinformatics tools. Although these in silico bioinformatic tools are useful to predict multi-genes associated with the pathophysiology of disorder, the in silico results are not strong enough to justify the functional significance of genes. Moreover, due to the very small amount of fetal chorionic cells that were obtained, we could not gain more confidence through further studies of gene expression in DS. Therefore, the functional roles of the potential pathways in our study need further characterization, possibly in cell lines or tissues relevant to specific DS phenotypes. These potential pathways with epigenetic changes in DS remain to be further elucidated. Furthermore, this study was limited by its small sample size and no adjustment for confounders. DNA methylation could be affected by various environmental and clinical factors. Therefore, future, larger studies are needed to substantiate our findings. Despite the limitations of this study, our findings warrant further studies addressing DNA methylation of the whole genome associated with the pathogenesis of DS. Various further studies might support the functional significance of our new insight into the pathophysiology of DS.

\section{Conclusion}

To our knowledge, this is the first study to perform high-throughput methylome profiling of the chorionic villi cells of DS fetuses using MC-seq. Various CpG sites across the whole genome were differentially methylated between euploid and DS fetuses, and most were hypermethylated in DS. Furthermore, our results indicate that 
many biological pathways implicated in DS and its complications may be regulated by genes with DMRs. Therefore, the current study provides a variety of information that may give a better understanding of epigenetic modulations in whole genome of DS.

\section{Material and methods}

\section{Study subjects}

The Institutional Review Board of Cheil General Hospital approved this study (\#CGH-IRB-2016-5). Singleton pregnant women who attended antenatal care at the hospital's Department of Obstetrics and Gynecology between June 2015 and May 2017 were enrolled for this study. Written informed consent was obtained from all patients under the approval from the IRB. Chorionic villi cells from subjects were obtained in the first trimester of pregnancy (12 $\sim 13$ weeks of gestation, Additional file 1: Table S1) and stored in liquid nitrogen until analysis. The gestational age of each fetus was determined by ultrasonography.

Chromosomal analyses using the GTG banding method were performed to determine the karyotypes of fetal chorionic villi cells. All DS samples were a complete extra copy of HSA21 (47, XX, +21 or $47, \mathrm{XY},+21)$ and all controls showed normal karyotypes (46, XX or 46, XY) (Additional file 1: Table S1). The causes for chromosomal analyses were maternal advanced age and increased nuchal translucency (Additional file 1: Table S1). The cases and controls were matched for the gender ratio of fetuses. The genomic DNA of each sample was extracted from chorionic villi cells using the QIAamp DNA Mini Kit (Qiagen, catalog no. 51304), according to the manufacturer's instructions.

\section{High-throughput methylome profiling using MC-seq}

Standard DNA methylation region capture libraries were generated using the SureSelect Methyl-Seq Target Enrichment protocol (Agilent) for a paired-end sequencing library (ver. B.3, June 2015; Illumina) with $3 \mu \mathrm{g}$ genomic DNA. In all cases, the SureSelect Human Methyl-Seq probe set (Agilent, catalog no. 5190-4662) was used. The quantification of DNA and the DNA quality was done using measured by PicoGreen assay kit (Thermo Fisher Scientific, catalog no. P7589) and Nanodrop spectrophotometer (NanoDrop Technologies, catalog no. ND2000), respectively.

Fragmentation of the $3 \mu \mathrm{g}$ of genomic DNA was performed using adaptive focused acoustic technology (AFA; Covaris, catalog no. 500219) to a target peak size of 150-200 bp. Briefly, the eight microTUBE Strips were loaded into the tube holder of the ultrasonicator and shear the DNA using the following settings: mode, frequency sweeping; duty cycle, $10 \%$; intensity, 5 ; cycles per burst, 200; duration, $60 \mathrm{~s} \times 6$ cycles; temperature, $4{ }^{\circ} \mathrm{C} \sim$ $7{ }^{\circ} \mathrm{C}$. The fragmented DNA is repaired, an "A" is ligated to the 3' end, SureSelect Methyl-Seq Methylated Adapter are then ligated to the fragment. Once ligation was assessed, the adapter ligated product was PCRamplified. The final purified product such as the methylated adapter-ligated DNA was then quantified according to the qPCR Quantification Protocol Guide and qualified using the TapeStation DNA screen tape D1000 (Agilent, catalog no. 5067-5582).

For DNA methylation region capture, 350 ng of DNA library was mixed with hybridization buffers, blocking mixes, RNase block, and $5 \mu \mathrm{l}$ of the SureSelect All DNA methylation region Capture Library, according to the standard SureSelect Methyl-Seq Target Enrichment protocol (Agilent). Hybridization to the capture bait was conducted at $65{ }^{\circ} \mathrm{C}$ using a heated thermal cycler lid option at $105{ }^{\circ} \mathrm{C}$ for $24 \mathrm{~h}$ with a PCR machine. Target captured DNAs were treated with bisulfite using the EZ DNA Methylation-Gold Kit (Zymo Research, catalog no. D5005), subjected to eight PCR cycles to enrich adaptor added fragments, and six PCR cycles to add multiplexing barcodes. The captured DNA was then amplified. The final purified product was quantified using the aforementioned qPCR Quantification Protocol Guide and qualified using the TapeStation DNA screen tape D1000 (Agilent, catalog no. 5067-5582). Sequencing was done using the HiSeq $^{\text {ti }} 2500$ platform (Illumina, catalog no. SY-401-2501).

\section{Data processing and methylation profile calling}

The quality of the paired-end raw reads generated from sequencing was checked using FastQC software (version 0.11.5). Before starting the analysis, Trimmomatic (version 0.32) was used to remove adapter sequences and bases with a base quality $<3$ from the end reads. Also, using the sliding window trim method, bases that did not qualify for window size $=4$, and mean quality $=15$ were removed. Reads with a minimum length of $36 \mathrm{bp}$ were removed to produce cleaned data. The cleaned reads were aligned to the Homo sapiens genome (UCSC hg19) using the bisulfite sequencing MAPping program (BSMAP; version 2.90 parameter set $-n 1-r$ ) based on the Short Oligo Alignment program (SOAP), which allowed only uniquely mapped reads. Mapped data in SAM file format were sorted and indexed using SAMtools (version 1.2). PCR duplicates were removed with sambamba (version 0.5.9).

The methylation level was determined with the methratio.py feature in the BSMAP program [37]. The methylation ratio of every single cytosine located in the Agilent SureSelect target region satisfying higher than 10 CT count was reliable generally as a methylation call. For the regions covered by both ends of a read pair, only one read was used to call methylation. The resulting coverage profiles were summarized as \# of C/effective 
CT counts for each of the three-sequence context (CG, $\mathrm{CHG}$, and $\mathrm{CHH}$ ).

\section{Identification of DMCs and DMRs}

The methylation level at each base of $\mathrm{CpG}$ was normalized with median scaling normalization. An independent $t$ test was used to assess the significance of methylation differences between the two groups for five comparison pair. The $p$ values were corrected using the Benjamini and Hochberg false discovery rate (FDR) method to control false-positive results from multiple testing as our prior study [10]. The principal component analysis revealed the separation of samples based on disease status (normal or DS) but not fetal gender, as a prior study [15].

DMCs were determined by filtering each region associated with |delta_mean $\mid \geq 0.3$, independent $t$ test $p$ values $<0.05$, and FDR $<0.05$. DMRs were defined as contiguous regions of any length containing $\geq 3$ DMCs. Hierarchical clustering analysis also was performed using complete linkage and Euclidean distance as a measure of similarity to display the methylation level of samples for significant CpGs, which satisfied at least one more comparison pair. The heatmap was plotted automatically by a centroid linkage using the centered absolute correlation in the similarity metric. All data analysis and visualization of the differentially methylated result was conducted using R 3.3.1 (www.r-project.org) and Statistical Package for Social Sciences 12.0 (SPSS Inc.).

\section{Comparison of DS fetal brain}

Microarray data for the brain of DS and control subjects were downloaded from the Gene Expression Omnibus (GEO, http://www.ncbi.nlm.nih.gov/geo/). A publicly available microarray dataset of human DS fetal brains (GEO \#GSE73747) was used. The dataset was reanalyzed according to the aforementioned DMC and DMR criteria. We used data of 61 fetal brain samples (36 normal fetal brains, 25 DS fetal brains).

\section{Methylation-specific quantitative real-time PCR of DMRs}

We confirmed the methylation level of the MC-seq data using methylation-specific quantitative real-time PCR. The genomic DNA of each sample was isolated from the chorionic villi cells of normal $(n=10)$ and DS $(n=8)$ fetuses using the QIAamp DNA Mini Kit (Qiagen, catalog no. 51304). Samples that had been used for the MC-seq were also included (normal DS $=5: 4)$. DNA samples (10 ng) were treated with Acil (New England Biolabs, cata$\log$ no. R0551L) as the methylation-specific restriction enzyme (MSRE) according to the manufacturer's instructions, and then concentrated to $40 \mu \mathrm{L}$ using the DNA Clean and Concentrator (Zymo Research, catalog no. D4031). Through this process, unmethylated DNA including the recognition sequence of MSRE was cleaved. The MSRE-treated DNA and untreated DNA were amplified by real-time PCR. The sequences of PCR primers used and PCR conditions are presented in Additional file 1: Table S8.

For the analysis of methylation levels of DMRs, the delta $(\Delta)$ threshold cycle $(\mathrm{Ct})$ value was calculated as $\Delta \mathrm{Ct}=\mathrm{Ct}_{\mathrm{MSRE}}-\mathrm{Ct}_{\text {input }}$. Input indicates the level of the target gene in whole genome without MSRE treatment and MSRE indicates the level of the methylated target gene that remained after MSRE treatment. Therefore, the $\Delta \mathrm{Ct}$ value represented the methylation level of the target gene. The smaller the $\Delta \mathrm{Ct}$ value, the higher the methylation level of the target gene. The $p$ value of the study subjects was analyzed using the Mann-Whitney $U$ test and a $p$ value $<0.05$ was considered statistically significant. Statistical analyses were performed using the Statistical Package for Social Sciences 12.0 (SPSS Inc.).

\section{Functional annotation of DMR genes}

The lists of genes with DMRs were submitted to a functional annotation tool provided by WebGestalt (http:// www.webgestalt.org/webgestalt_2013/). Gene ontology (GO) analysis and disease-associated gene analysis were performed according to criteria of a statistic hypergeometric test with a significance level of adj $P<0.05$, a Benjamini and Hochberg multiple test adjustment, and a minimum of three genes [10].

\section{Supplementary information}

Supplementary information accompanies this paper at https://doi.org/10. 1186/s13148-019-0756-4

Additional file 1: Table S1. Clinical characteristics. Table S2. MC-seq performance of each sample. Table S3. CpG sites of each sample covered by MC-seq. Table S4. Methylation levels of DMCs in the functional genomic regions. Table S5. Methylation levels of DMCs in the fetal normal and DS brain. Table S6. Genes with DMRs. Table S7. Functional region and chromosomal location of genes with DMRs. Table S8. Primer sequences and PCR condition for methylation specific quantitative realtime PCR .

\section{Abbreviations}

CGl: CpG islands; Ct: Threshold cycle; DMCs: Differentially methylated CpG sites; DMRs: Differentially methylated regions; DS: Down syndrome; GO: Gene ontology; HSA21: Human chromosome 21; MC-Seq: Methyl-capture sequencing; MSRE: Methylation-specific restriction enzyme

\section{Acknowledgements}

We thank the following physicians who took the time and effort to contribute to this study: Ju Yeon Park, Hyun Jin Kim, Yeon Woo Lee, Ah Rum Oh, and Shin Yeong Lee.

\section{Authors' contributions}

$J H L, Y H C$, and HMR contributed to conception and design. JHL, YJK, BYL, and JWK helped in the experimental part, analysis, and interpretation of data. $J H L, Y J H$, and BYL prepared the manuscript. YJH, JHC, MYK, MHK, and HMR collected the sample and maintained the patient database. YHC and HMR are the principal investigators of the project. All authors read and approved the final manuscript. 


\section{Funding}

This work was funded by grants (HI16C0628) from the Korea Health Technology R\&D Project through the Korea Health Industry Development Institute (KHIDI), funded by the Ministry of Health and Welfare, Republic of Korea. The funder had no role in study design, data collection and analysis, decision to publish, or preparation of the manuscript.

\section{Availability of data and materials}

Not applicable.

\section{Ethics approval and consent to participate}

The Institutional Review Board (IRB) at Cheil General Hospital approved all research included this study (\#CGH-IRB-2016-5). Written informed consent was obtained from all participants under the approval from the IRB.

\section{Consent for publication}

Applicable.

\section{Competing interests}

All authors declare that they have no competing interests.

\section{Author details}

${ }^{1}$ Center for Biomarker Research and Precision Medicine, CHA Advanced Research Institute, Gyeonggi-do, Republic of Korea. ${ }^{2}$ Department of Medical Genetics, College of Medicine, Hanyang University, 222, Wangsimni-ro, Seongdong-gu, Seoul 04763, Republic of Korea. ${ }^{3}$ SD Genomics Co., Ltd., Seoul, Republic of Korea. ${ }^{4}$ Department of Obstetrics and Gynecology, CHA Gangnam Medical Center, CHA University, Seoul, Republic of Korea. ${ }^{5}$ Department of Obstetrics Gynecology, Mizmedi Hospital, Seoul, Republic of Korea. 'Laboratory of Medical Genetics, Medical Research Institute, Cheil General Hospital and Women's Healthcare Center, Seoul, Republic of Korea. ${ }^{7}$ Department of Obstetrics and Gynecology, CHA Bundang Medical Center, CHA University, 59, Yatap-ro, Bundang-gu, Seongnam-si, Gyeonggi-do 13496, Republic of Korea.

Received: 22 July 2019 Accepted: 6 October 2019

Published online: 04 December 2019

\section{References}

1. Do C, Xing Z, Yu YE, Tycko B. Trans-acting epigenetic effects of chromosomal aneuploidies: lessons from Down syndrome and mouse models. Epigenomics. 2017;9:189-207.

2. www.who.int/genomics/public/geneticdiseases/en/index1.htm

3. Ostermaier KK. Down syndrome: Clinical features and diagnosis. www. uptodate.com/contents/down-syndrome-clinical-features-and-diagnosis

4. FitzPatrick DR, Ramsay J, McGill NI, Shade M, Carothers AD, Hastie ND. Transcriptome analysis of human autosomal trisomy. Hum Mol Genet. 2002;11:3249-56.

5. Aït Yahya-Graison E, Aubert J, Dauphinot L, Rivals I, Prieur M, Golfier G, et al. Classification of human chromosome 21 gene-expression variations in Down syndrome: impact on disease phenotypes. Am J Hum Genet. 2007;81:475-91.

6. Kahlem P, Sultan M, Herwig R, Steinfath M, Balzereit D, Eppens B, et al. Transcript level alterations reflect gene dosage effects across multiple tissues in a mouse model of down syndrome. Genome Res. 2004;14:1258-67.

7. Lyle R, Gehrig C, Neergaard-Henrichsen C, Deutsch S, Antonarakis SE. Gene expression from the aneuploid chromosome in a trisomy mouse model of down syndrome. Genome Res. 2004;14:1268-74.

8. Letourneau A, Santoni FA, Bonilla X, Sailani MR, Gonzalez D, Kind J, et al. Domains of genome-wide gene expression dysregulation in Down's syndrome. Nature. 2014:508:345-50.

9. Sailani MR, Santoni FA, Letourneau A, Borel C, Makrythanasis P, Hibaoui Y, et al. DNA-methylation patterns in trisomy 21 using cells from monozygotic twins. PLoS One. 2015;10:e0135555.

10. Lim JH, Han YJ, Kim HJ, Kwak DW, Park SY, Chun SH, et al. Genome-wide gene expression analysis in the placenta from fetus with trisomy 21. BMC Genomics. 2017;18:720.

11. Greaves IK, Gonzalez-Bayon R, Wang L, Zhu A, Liu PC, Groszmann M, et al. Epigenetic changes in hybrids. Plant Physiol. 2015;168:1197-205.
12. Brunner AL, Johnson DS, Kim SW, Valouev A, Reddy TE, Neff NF, et al Distinct DNA methylation patterns characterize differentiated human embryonic stem cells and developing human fetal liver. Genome Res. 2009:19:1044-56.

13. Lu J, Mccarter M, Lian G, Esposito G, Capoccia E, Delli-Bovi LC, et al. Global hypermethylation in fetal cortex of Down syndrome due to DNMT3L overexpression. Hum Mol Genet. 2016;25:1714-27.

14. El Hajj N, Dittrich M, Böck J, Kraus TF, Nanda I, Müller T, et al. Epigenetic dysregulation in the developing Down syndrome cortex. Epigenetics. 2016;11:563-78.

15. Jin S, Lee YK, Lim YC, Zheng Z, Lin XM, Ng DP, et al. Global DNA hypermethylation in Down syndrome placenta. PLoS Genet. 2013;9: e1003515.

16. Serra-Juhé C, Cuscó I, Homs A, Flores R, Torán N, Pérez-Jurado LA. DNA methylation abnormalities in congenital heart disease. Epigenetics. 2015 10:167-77.

17. Jones MJ, Farré P, McEwen LM, Macisaac JL, Watt K, Neumann SM, et al. Distinct DNA methylation patterns of cognitive impairment and trisomy 21 in Down syndrome. BMC Med Genomics. 2013;6:58.

18. Kerkel K, Schupf N, Hatta K, Pang D, Salas M, Kratz A, et al. Altered DNA methylation in leukocytes with trisomy 21. PLoS Genet. 2010;6:e1001212.

19. Horvath S, Garagnani P, Bacalini MG, Pirazzini C, Salvioli S, Gentilini D, et al. Accelerated epigenetic aging in Down syndrome. Aging Cell. 2015;14:491-5.

20. Teh AL, Pan H, Lin X, Lim Yl, Patro CP, Cheong CY, et al. Comparison of methyl-capture sequencing vs. Infinium 450K methylation array for methylome analysis in clinical samples. Epigenetics. 2016;11:36-48.

21. Gupta AK, Holzgreve W, Huppertz B, Malek A, Schneider H, Hahn S. Detection of fetal DNA and RNA in placenta-derived syncytiotrophoblast microparticles generated in vitro. Clin Chem. 2004;50:2187-90.

22. www.ncbi.nlm.nih.gov/gene/1375.

23. www.genecards.org/cgi-bin/carddisp.pl?gene=SHROOM1

24. Lee C, Scherr HM, Wallingford JB. Shroom family proteins regulate gammatubulin distribution and microtubule architecture during epithelial cell shape change. Development. 2007;134:1431-41.

25. Bonin CA, Lewallen EA, Baheti S, Bradley EW, Stuart MJ, Berry DJ, et al. Identification of differentially methylated regions in new genes associated with knee osteoarthritis. Gene. 2016:576:312-8.

26. www.ncbi.nlm.nih.gov/gene/54897.

27. Charpentier MS, Christine KS, Amin NM, Dorr KM, Kushner EJ, Bautch VL, et al. CASZ1 promotes vascular assembly and morphogenesis through the direct regulation of an EGFL7/RhoA-mediated pathway. Dev Cell. 2013:25:132-43

28. Visootsak J, Hess B, Bakeman R, Adamson LB. Effect of congenital heart defects on language development in toddlers with Down syndrome. J Intellect Disabil Res. 2013;57:887-92.

29. Versacci P, Di Carlo D, Digilio MC, Marino B. Cardiovascular disease in Down syndrome. Curr Opin Pediatr. 2018;30:616-22.

30. Sojka S, Amin NM, Gibbs D, Christine KS, Charpentier MS, Conlon FL. Congenital heart disease protein 5 associates with CASZ1 to maintain myocardial tissue integrity. Development. 2014;141:3040-9.

31. Liu Z, Li W, Ma X, Ding N, Spallotta F, Southon E, et al. Essential role of the zinc finger transcription factor Casz1 for mammalian cardiac morphogenesis and development. J Biol Chem. 2014;289:29801-16.

32. www.genecards.org/cgi-bin/carddisp.pl?gene $=$ C11 orf95.

33. Parker M, Mohankumar KM, Punchihewa $C$, Weinlich $R$, Dalton JD, Li $Y$, et al C11 orf95-RELA fusions drive oncogenic NF-KB signalling in ependymoma. Nature. 2014:506:451-5.

34. Rickert CH, Göcke H, Paulus W. Fetal ependymoma associated with Down's syndrome. Acta Neuropathol. 2002:103:78-81.

35. Biswas A, Puri T, Goyal S, Kumar S, Sharma A, Paliwal P, et al. Spinal myxopapillary ependymoma with Down syndrome: exploring an unusual association. J Pediatr Hematol Oncol. 2010;32:e38-41.

36. www.genecards.org/cgi-bin/carddisp.pl?gene=KIAA1875.

37. Xi Y, Li W. BSMAP: whole genome bisulfite sequence MAPping program. BMC Bioinformatics. 2009;10:232

\section{Publisher's Note}

Springer Nature remains neutral with regard to jurisdictional claims in published maps and institutional affiliations. 